\title{
Ipsilateral hemiparesis: the forgotten history of this paradoxical neurological sign
}

\author{
Rodrigo Carrasco-Moro, MD, Ines Castro-Dufourny, MD, PhD, ${ }^{2}$ \\ Juan S. Martínez-San Millán, MD, PhD, ${ }^{3}$ Lidia Cabañes-Martínez, MD, ${ }^{4}$ and \\ José M. Pascual, MD, PhD 5
}

'Department of Neurosurgery, Ramón y Cajal University Hospital; ${ }^{2}$ Department of Endocrinology, Sureste University Hospital, Francisco de Vitoria University; Departments of ${ }^{3}$ Neuroradiology and ${ }^{4}$ Clinical Neurophysiology, Ramón y Cajal University Hospital; and ${ }^{5}$ Department of Neurosurgery, La Princesa University Hospital, Madrid, Spain

OBJECTIVE Establishing the neurological localization doctrine for the contralateral hemispheric control of motor functions in the second half of the 19th century, researchers faced the challenge of recognizing false localizing signs, in particular paradoxical or ipsilateral hemiparesis $(\mathrm{IH})$. Despite tremendous progress in current methods of neuroradiological and electrophysiological exploration, a complete understanding of this phenomenon has yet to be attained.

METHODS The authors researched the well-described cases of hemiparesis/hemiplegia ipsilateral to an intracranial lesion published in the scientific literature in the pre-MRI era (before 1980). A comprehensive review of the physiopathological mechanisms proposed for paradoxical hemiparesis throughout this period, as well as the pathological evidence substantiating them, is provided.

RESULTS A collection of 75 patients with hemiparesis/hemiplegia ipsilateral to the primary intracranial lesion reported between 1858 and 1979 were eligible for analysis. Most cases occurred in adults with supratentorial, slowly developing, extraparenchymatous mass lesions, such as neoplasms (38\%) or chronic subdural hematomas (36\%). Physiopathological theories proposed by the neurologists who investigated IH can be grouped into 4 major concepts: 1) lack of anatomical decussation of the corticospinal tract; 2) impaired functional activation of the contralateral hemisphere by the lesioned dominant hemisphere through the callosal connections; 3) Kernohan's notch phenomenon, or mechanical injury of the contralateral cerebral peduncle against the free edge of the tentorium; and 4) cerebrovascular dysfunction involving the contralateral hemisphere owing to kinking and mechanical flattening of the carotid artery contralateral to the primary intracranial lesion.

CONCLUSIONS IH represents a still underdiagnosed paradoxical neurological phenomenon. With the aid of modern neuroradiological and neurophysiological methods, Kernohan's peduncle notch mechanism has been confirmed to cause $\mathrm{IH}$ in many of the cases reported in recent decades. Nevertheless, alternative functional and/or vascular mechanisms must be investigated further for unexplained $\mathrm{IH}$ cases, in particular for transitory $\mathrm{IH}$ without evidence of peduncle injury. The historical theories reviewed in this paper represent a conceptual framework that may be helpful for this purpose. https://thejns.org/doi/abs/10.3171/2019.6.FOCUS19337

KEYWORDS diaschisis; ipsilateral hemiparesis; Kernohan-Woltman notch phenomenon; history of neurology; paradoxical hemiparesis; tentorial incisure

In the vast majority of cases, cerebral palsy is crossed in relation to the lesions that determine it, that is, lesions of the right hemisphere of the brain give rise to paralysis on the left side of the body, and lesions of the left hemisphere to paralysis of the right side. This is the law. It has been established in innumerable observations and finds its natural explanation in the fact of the decussation of the pyramidal bundles at the level of the Mistichelli intercross. Well! This law is not absolute. There are some observations of hemiplegia on one side of the body caused by cerebral hemisphere lesions on the same side...

- Jean-Martin Charcot and Albert Pitres, 189511

Modern intracranial surgery began in the closing decades of the 19th century, stimulated by the development of localizationist theories that, along with progress in the

ABBREVIATIONS ADC = apparent diffusion coefficient; $I H=$ ipsilateral hemiparesis. ACCOMPANYING EDITORIAL DOI: 10.3171/2019.6.FOCUS19501.

SUBMITTED April 30, 2019. ACCEPTED June 12, 2019.

INCLUDE WHEN CITING DOI: 10.3171/2019.6.FOCUS19337. 
fields of antisepsis and anesthesia, allowed intrepid surgeons to design accurate approaches to cerebral lesions. Nevertheless, expanding intracranial lesions occasionally produced focal neurological signs not directly related to the location of the mass. These paradoxical clinical findings, termed "false localizing signs" for James Collier's studies, ${ }^{13}$ disconcerted both clinicians and surgeons as they frequently led to wrong-site exploratory surgical procedures. Among false localizing signs, ipsilateral hemiparesis $(\mathrm{IH})$ relative to an intracranial mass - widely known as Kernohan's notch phenomenon-remains one of the most enigmatic. Despite the use of modern neuroradiological and electrophysiological exploration methods, the intrinsic mechanisms leading to the development of $\mathrm{IH}$ have not been fully clarified. ${ }^{45}$ In this work, we performed a thorough analysis of a cohort of historical reports of patients with verified IH published before the advent of modern neuroradiology. Additionally, we provide a comprehensive review of the physiopathological hypotheses put forward for this intriguing sign by prominent figures of neurology and neurosurgery in the 19th and 20th centuries. These early clinical observations and hypotheses, rarely cited in modern scientific literature, may contribute to an appropriate understanding of this paradoxical clinical phenomenon.

\section{Methods}

We conducted a thorough review of well-described cases of IH published in the scientific literature in the pre-MRI era (prior to the 1980s). This survey involved reports in official medical journals and in specialized texts, monographs, and doctoral dissertations. The initial search included all articles shown in the PubMed, MEDLINE, and Scopus databases after entering the keywords "ipsilateral hemiparesis," "Kernohan's notch," and "KernohanWoltman notch phenomenon." Reference lists from the selected articles were scrutinized for a systematic retrospective retrieving of older articles/monographs. From these documents, all individual cases of IH providing clinical-surgical and/or clinical-gross pathological verification of the intracranial lesion giving rise to the ipsilateral motor deficit were included in this study. A comprehensive review of the physiopathological mechanisms proposed for paradoxical hemiparesis and the pathological evidence substantiating them in this set of scientific studies was carried out.

\section{Results}

Seventy-five IH patients reported on between 1858 and 1979 were included in this analysis. Table 1 features a summary of the data corresponding to the fundamental variables selected and analyzed in this cohort. Most IH cases occurred in adult patients (95.7\%) following a time course that was subacute or chronic in $73.6 \%$. The main intracranial pathologies causing $\mathrm{IH}$ were neoplasms $(38.6 \%)$ and chronic subdural hematomas (36\%). These lesions involved the supratentorial compartment in $94.6 \%$. A high proportion of intracranial neoplasms corresponded to extraparenchymatous tumors. The diagnosis of the associated intracranial pathology was made at autopsy in 32 patients, 7 of whom had undergone an exploratory craniotomy on the wrong side. In this subgroup the morphology of pyramidal decussation at the bulbomedullary junction was examined in 8 specimens, 3 of which displayed uncrossed corticospinal tracts. In another 3 cases, a structural lesion involving the contralateral cerebral peduncle was noted. In the remaining 43 patients in whom an autopsy was not performed, an accurate preoperative topographical diagnosis of the mass could be achieved with the aid of ventriculography and/or angiography. In this latter subgroup, complete or partial recovery from the motor deficit occurred in all but 3 patients, who died from surgical complications or disease progression. In 2 of Dupuis-Leveau's patients, IH reappeared when the subdural hematoma recurred after the surgical procedure, and 2 additional patients presented with paradoxical hemiparesis as a consequence of the development of a new contralateral subdural hematoma. ${ }^{21}$

\section{Discussion}

At the turn of the 20th century, most clinicians who faced the dilemma of IH considered this symptom exceedingly rare. A variety of terms had been used for this phenomenon, and among them were the following: homolateral, ipsilateral, direct, and paradoxical hemiparesis. ${ }^{6,14,34}$ Most authors unanimously emphasized the importance of $\mathrm{IH}$ as a confusing sign that might lead to an exploratory trepanation on the wrong side (Table 1). Furthermore, prior to the introduction of ventriculography and angiography for localizing intracranial pathologies, the confirmation of IH could only be established during autopsy. The physiopathology of this phenomenon seriously disconcerted neurologists, who developed numerous hypotheses, some of them rather speculative, based on the existing clinicoanatomical evidence available. ${ }^{34} \mathrm{~A}$ thorough analysis of the verified historical IH cases has allowed us to group these hypotheses into 4 major theoretical concepts, which will be separately analyzed in depth (Table 2 ).

\section{Lack of Anatomical Decussation of the Corticospinal Tract}

In the 1870s, a series of physiological and pathological breakthroughs contributed to debunking the theory of functional homogeneity in the human brain, largely defended by neurologists up to that time. The work by French neurologists Albert Pitres (1848-1928) and his mentor Jean-Martin Charcot (1825-1893) noticeably challenged this old paradigm by localizing the brain cortical regions involved in governing motor functions. ${ }^{11}$ Pitres and Charcot deemed highly questionable the common theories supporting the impairment of the contralateral corticospinal tract in motor deficits, a conclusion derived from the assessment of patients with brain tumors or injuries without a clear topographical demarcation. They instead adapted the novel clinicoanatomical method to the field of neurology and investigated only the neurological signs observed in patients with brain ischemia or hemorrhage showing an irrefutable definition of brain injury at autopsy. ${ }^{11}$ Although limited to a single case, their research provided solid clinicopathological evidence for the lack of pyramidal tract decussation as an explanation for IH (Fig. 1), a hypothesis 
TABLE 1. Summary of data for the cohort of well-described patients with IH reported in the pre-MRI era (1858-1979)

\begin{tabular}{|c|c|c|c|c|c|c|c|}
\hline $\begin{array}{l}\text { Case } \\
\text { No. }\end{array}$ & Authors \& Year* & $\begin{array}{l}\text { Age (yrs), } \\
\text { Sex }\end{array}$ & $\begin{array}{l}\text { Time Onset/ } \\
\text { Etiology }\end{array}$ & Motor Deficit & $\begin{array}{c}\text { Additional Neurological } \\
\text { Symptoms }\end{array}$ & Pathological/Surgical Evidence & Outcome \\
\hline 1 & $\begin{array}{l}\text { Brown-Séquard, } \\
1858\end{array}$ & $41, \mathrm{~F}$ & Chr/tumor & Lt hemiparesis & $\begin{array}{l}\text { Lt CN V neuralgia, dys- } \\
\text { phagia, It hypoestesia }\end{array}$ & $\begin{array}{l}\text { Lt middle fossa-CPA extraaxial } \\
\text { giant tumor }\end{array}$ & Death \\
\hline 2 & Couty, 1877 & $\mathrm{NA}, \mathrm{M}$ & SA/infection & Lt hemiplegia & Sz, ataxia & $\begin{array}{l}\text { Tuberculous meningitis; It } \\
\text { cerebellar abscess }\end{array}$ & Death \\
\hline 3 & Blaise, 1882 & NA & NA & Rt hemiplegia & NA & Rt putaminal hemorrhage $(X)$ & Death \\
\hline 4 & $\begin{array}{l}\text { Charcot \& Pitres, } \\
1895\end{array}$ & $66, \mathrm{M}$ & Ac/infection & Lt hemiplegia & Motor aphasia & $\begin{array}{l}\text { Lt frontal infarction, It CST } \\
\text { degeneration (Ж) }\end{array}$ & Death \\
\hline 5 & $\begin{array}{l}\text { Vanhenverswyn, } \\
1888\end{array}$ & $55, \mathrm{~F}$ & Ac/hematoma & Lt hemiparesis & HICP, anisocoria, coma & Lt frontoparietal cSDH & Death \\
\hline 6 & Lederhose, $1896^{6}$ & $38, \mathrm{M}$ & $\mathrm{SA} / \mathrm{TBI}$ & Rt hemiplegia & HICP, rt mydriasis, coma & $\begin{array}{l}\text { Rt EDH \& brain contusions (It } \\
\text { trephination negative) }\end{array}$ & Death \\
\hline 7 & Lederhose, $1896^{6}$ & $33, \mathrm{M}$ & $\mathrm{SA} / \mathrm{TBI}$ & Lt hemiplegia & HICP, It mydriasis, coma & $\begin{array}{l}\text { Lt EDH \& contusions (rt crani- } \\
\text { otomy negative) }\end{array}$ & Death \\
\hline 8 & Pineles, $1896,{ }^{6} \mathrm{c} 1$ & $\mathrm{NA}, \mathrm{M}$ & SA/hematoma & Rt hemiplegia & None & Rt cSDH (X) & Death \\
\hline 9 & Pineles, $1896,{ }^{6}$ c2 & NA & $\mathrm{Ac} / \mathrm{TBI}$ & Rt hemiplegia & Coma & $\begin{array}{l}\text { Rt hemispheric SAH (It trephina- } \\
\text { tion negative) }\end{array}$ & Death \\
\hline 10 & Pineles, $1896,{ }^{6}$ c3 & $60, \mathrm{M}$ & $\mathrm{Ac} / \mathrm{TBI}$ & Rt hemiplegia & None & $\begin{array}{l}\text { Rt trephination positive for } \\
\quad \text { brownish fluid (impact zone) }\end{array}$ & Recovery \\
\hline 11 & Pineles, $1896,{ }^{6}$ c4 & $4, M$ & NA/tumor & Rt hemiplegia & HICP, ataxia & Rt parietal gliosarcoma & Death \\
\hline 12 & Zenner, $1898^{6}$ & $33, \mathrm{M}$ & Chr/tumor & Lt hemiparesis & HICP, Sz, S & $\begin{array}{l}\text { Lt peri-rolandic extraaxial mass, } \\
\text { It CST degeneration }(Ж)\end{array}$ & Death \\
\hline 13 & Bernex, $1904^{6}$ & $52, \mathrm{~F}$ & Ac/hematoma & Rt hemiplegia & Motor aphasia & Rt basal ganglia hemorrhage & Death \\
\hline 14 & $\begin{array}{l}\text { Dupré \& Camus, } \\
1905\end{array}$ & $43, M$ & Ac/infection & Lt hemiplegia & $\begin{array}{l}\text { Aphasia, It mydriasis, } \\
\text { confusion }\end{array}$ & $\begin{array}{l}\text { Lt peri-rolandic infarction, rt } \\
\text { CST atrophy }(Ж)\end{array}$ & Death \\
\hline 15 & Pic, 1906 & $64, \mathrm{M}$ & Ac/hematoma & Rt hemiplegia & Dysphasia & Rt occipital hemorrhage & Death \\
\hline 16 & Joltrain, $1906^{6}$ & $54, \mathrm{M}$ & $\mathrm{SA} / \mathrm{TBI}$ & Lt hemiplegia & HICP, Sz, stupor & Lt aSDH & Death \\
\hline 17 & Burley, $1906^{6}$ & $7, \mathrm{M}$ & SA/infection & Rt hemiplegia & $\mathrm{HICP}, \mathrm{Sz}$ & Rt hemispheric abscess & Death \\
\hline 18 & Oppenheim, $1906^{6}$ & $15, M$ & SA/tumor & Lt hemiparesis & $\begin{array}{l}\text { HICP, Sz, It ataxia, It CN } \\
\text { VII \& lower CN paresis }\end{array}$ & $\begin{array}{l}\text { Lt petrous extraaxial mass } \\
\text { surgically treated }\end{array}$ & Death \\
\hline 19 & Clunet, $1908^{6}$ & $68, \mathrm{M}$ & Ac/hematoma & Rt hemiplegia & NA & $\begin{array}{l}\text { Rt hemispheric \& ventricular } \\
\text { hemorrhage }\end{array}$ & Death \\
\hline 20 & $\begin{array}{l}\text { Babinski \& Clunet, } \\
1908\end{array}$ & $43, M$ & Chr/tumor & Rt hemiparesis & HICP, stupor & $\begin{array}{l}\text { Rt hemispheric extraaxial tumor } \\
\qquad(\mathrm{X})\end{array}$ & Death \\
\hline 21 & Ascenzi, 1908 & $64, \mathrm{M}$ & Chr/hematoma & $\begin{array}{l}\text { Bilateral hemi- } \\
\text { paresis }\end{array}$ & Tremor, stupor & $\begin{array}{l}\text { Lt paracallosal (genu) hemor- } \\
\text { rhagic cyst }\end{array}$ & Death \\
\hline 22 & Binet, 1909 & $18, F$ & SA/infection & Lt hemiplegia & Lt oto-mastoiditis & Lt temporal abscess drained & Recovery \\
\hline 23 & Claude et al., 1910 & $43, F$ & Chr/tumor & Rt hemiplegia & HICP, It hemianopsia & $\begin{array}{l}\text { Rt occipital intraaxial tumor } \\
\text { surgically treated }\end{array}$ & Death \\
\hline 24 & Bonola, $1916^{14}$ & $22, \mathrm{M}$ & $\mathrm{Ac} / \mathrm{TBI}$ & Lt hemiplegia & Sz & $\begin{array}{l}\text { Lt frontal fracture surgically } \\
\text { treated }\end{array}$ & Unchanged \\
\hline 25 & $\begin{array}{l}\text { Demole de Ge- } \\
\text { nève, } 1918^{14}\end{array}$ & $42, \mathrm{M}$ & SA/tumor & Lt hemiplegia & Sz, coma & Lt frontal glioma $(X)$ & Death \\
\hline 26 & Giannuli, $1918^{14}$ & $59, \mathrm{~F}$ & Ac/hematoma & Lt hemiplegia & Lt CN III paresis, coma & Lt temporal hemorrhage (X) & Death \\
\hline 27 & Flatau, 1924 & $45, M$ & Chr/tumor & Lt hemiparesis & HICP, Sz & Lt frontal encapsulated tumor & Death \\
\hline 28 & Couronne, 1925 & $50, \mathrm{~F}$ & Ac/hematoma & Lt hemiplegia & Lt CN III paresis, coma & Lt aSDH & Death \\
\hline 29 & $\begin{array}{l}\text { Purves-Stewart, } \\
\text { 1927, c5 }\end{array}$ & $38, \mathrm{M}$ & Chr/tumor & Rt hemiparesis & $\begin{array}{l}\mathrm{HICP}, \mathrm{Sz} \text {, It CN VII } \\
\text { paresis }\end{array}$ & Rt precentral cystic sarcoma & Death \\
\hline 30 & $\begin{array}{l}\text { Purves-Stewart, } \\
\text { 1927, c10 }\end{array}$ & $28, F$ & Chr/tumor & Lt hemiparesis & HICP, Sz, stupor & $\begin{array}{l}\text { Lt frontal glioma surgically } \\
\text { treated }\end{array}$ & Death \\
\hline 31 & $\begin{array}{l}\text { Purves-Stewart, } \\
\text { 1927, c26 }\end{array}$ & $41, \mathrm{~F}$ & Chr/tumor & Lt hemiparesis & BT hemianopsia, S & $\begin{array}{l}\text { Lt middle fossa endothelioma } \\
\text { treated w/ bilateral DTC }\end{array}$ & Death \\
\hline
\end{tabular}


» CONTINUED FROM PAGE 3

TABLE 1. Summary of data for the cohort of well-described patients with IH reported in the pre-MRI era (1858-1979)

\begin{tabular}{|c|c|c|c|c|c|c|c|}
\hline $\begin{array}{l}\text { Case } \\
\text { No. }\end{array}$ & Authors \& Year* & $\begin{array}{l}\text { Age (yrs), } \\
\text { Sex }\end{array}$ & $\begin{array}{l}\text { Time Onset/ } \\
\text { Etiology }\end{array}$ & Motor Deficit & $\begin{array}{l}\text { Additional Neurological } \\
\text { Symptoms }\end{array}$ & Pathological/Surgical Evidence & Outcome \\
\hline 32 & $\begin{array}{l}\text { Groeneveld \& } \\
\text { Schaltenbrand, } \\
1927\end{array}$ & $43, M$ & Chr/tumor & Lt hemiparesis & $\begin{array}{r}\text { Rt limbs hyperkinetic } \\
\text { movements, HICP }\end{array}$ & Lt GSW meningioma (CPL) & Death \\
\hline 33 & $\begin{array}{l}\text { Kernohan \& Wolt- } \\
\text { man, } 1928\end{array}$ & $47, \mathrm{M}$ & NA/tumor & Lt hemiparesis & HICP, Sz, confusion & $\begin{array}{l}\text { Rt frontotemporal meningioma } \\
\quad(\mathrm{CPL})\end{array}$ & Death \\
\hline 34 & Torkildsen, $1937^{22}$ & $48, M$ & Chr/tumor & Lt hemiparesis & Motor aphasia & Lt frontal extraaxial mass & Death \\
\hline 35 & Ectors, 1945, c1 & $35, \mathrm{M}$ & Chr/tumor & Lt hemiparesis & HICP, anergia & $\begin{array}{l}\text { Lt frontal meningioma surgically } \\
\text { treated }\end{array}$ & Recovery \\
\hline 36 & Ectors, 1945, c2 & $46, F$ & Chr/tumor & Rt hemiparesis & HICP, anergia & $\begin{array}{l}\text { Rt frontal meningioma surgically } \\
\text { treated }\end{array}$ & Recovery \\
\hline 37 & Ectors, 1945, c3 & $53, F$ & Chr/tumor & $\begin{array}{l}\text { Rt hyperre- } \\
\text { flexia }\end{array}$ & HICP, anergia & $\begin{array}{l}\text { Rt frontal meningioma surgically } \\
\text { treated }\end{array}$ & Recovery \\
\hline 38 & Ectors, 1945, c4 & $45, M$ & Chr/tumor & Lt hemiparesis & HICP, stupor & $\begin{array}{l}\text { Lt hemispheric glioblastoma } \\
\text { (CPL) }\end{array}$ & Death \\
\hline 39 & $\begin{array}{l}\text { Ectors \& Heernu, } \\
\quad 1946\end{array}$ & $45, F$ & Chr/tumor & Lt hemiparesis & HICP, anergia & $\begin{array}{l}\text { Lt frontal meningioma surgically } \\
\text { treated }\end{array}$ & Recovery \\
\hline 40 & $\begin{array}{l}\text { Obrador Alcalde, } \\
1948\end{array}$ & $48, F$ & Chr/tumor & Lt hemiplegia & HICP, bradipsyquia & $\begin{array}{l}\text { Lt GSW meningioma surgically } \\
\text { treated }\end{array}$ & Recovery \\
\hline 41 & Ardito, 1954, c1 & $41, \mathrm{M}$ & SA/hematoma & Lt hemiparesis & Stupor & Lt cSDH surgically treated & Recovery \\
\hline 42 & Ardito, $1954, \mathrm{c} 2$ & $62, \mathrm{M}$ & SA/hematoma & Rt hemiparesis & HICP, confusion & Rt cSDH surgically treated & NA \\
\hline 43 & Ardito, 1954, c3 & $65, F$ & SA/hematoma & Rt hemiplegia & HICP, stupor & Rt cSDH surgically treated & Improvement \\
\hline 44 & Ardito, 1954, c4 & $40, M$ & SA/hematoma & Rt hemiparesis & HICP, stupor & Rt cSDH surgically treated & NA \\
\hline 45 & $\begin{array}{l}\text { Achslogh \& } \\
\quad \text { Macken, } 1959\end{array}$ & $55, \mathrm{M}$ & Chr/tumor & Rt hemiparesis & Sz, anergia & $\begin{array}{l}\text { Rt frontal meningioma surgically } \\
\text { treated }\end{array}$ & Recovery \\
\hline 46 & $\begin{array}{l}\text { Ectors \& Achslogh, } \\
\text { 1959, c2 }\end{array}$ & $44, \mathrm{M}$ & Chr/tumor & Rt hemiparesis & $\begin{array}{l}\text { Lt hemianopsia, rt CN III } \\
\text { palsy, HICP }\end{array}$ & $\begin{array}{l}\text { Rt frontotemporal meningioma } \\
\text { surgically treated }\end{array}$ & NA \\
\hline 47 & $\begin{array}{l}\text { Ectors \& Achslogh, } \\
\text { 1959, c4 }\end{array}$ & $39, F$ & SA/tumor & Lt hemiparesis & $\begin{array}{l}\text { HICP, rt CN VII palsy, } \\
\text { dysphasia }\end{array}$ & $\begin{array}{l}\text { Lt frontal meningioma surgically } \\
\text { treated }\end{array}$ & Recovery \\
\hline 48 & $\begin{array}{l}\text { Ectors \& Achslogh, } \\
\text { 1959, c5 }\end{array}$ & $45, M$ & Chr/tumor & Rt hemiparesis & HICP, stupor & $\begin{array}{l}\text { Rt frontal astrocytoma surgically } \\
\text { treated }\end{array}$ & Recovery \\
\hline 49 & $\begin{array}{l}\text { Peyser \& Doron, } \\
\text { 1966, c1 }\end{array}$ & $63, \mathrm{M}$ & Chr/tumor & Lt hemiparesis & HICP, rt hemianopsia & $\begin{array}{l}\text { Lt parietotemporal meningioma } \\
\text { surgically treated }\end{array}$ & Improvement \\
\hline 50 & $\begin{array}{l}\text { Peyser \& Doron, } \\
\text { 1966, c2 }\end{array}$ & $38, \mathrm{M}$ & Chr/tumor & Lt hemiparesis & HICP, confusion & $\begin{array}{l}\text { Lt GSW meningioma surgically } \\
\text { treated }\end{array}$ & Recovery \\
\hline 51 & $\begin{array}{l}\text { Peyser \& Doron, } \\
\text { 1966, c3 }\end{array}$ & $35, F$ & Chr/tumor & Lt hemiparesis & $\mathrm{HICP}, \mathrm{Sz}$ & $\begin{array}{l}\text { Lt frontal glioma surgically } \\
\text { treated }\end{array}$ & Recovery \\
\hline 52 & $\begin{array}{l}\text { Peyser \& Doron, } \\
\text { 1966, c4 }\end{array}$ & $54, \mathrm{M}$ & SA/hematoma & Lt hemiparesis & Confusion, apathy & Lt cSDH surgically treated & Recovery \\
\hline 53 & $\begin{array}{l}\text { Peyser \& Doron, } \\
\text { 1966, c5 }\end{array}$ & $58, \mathrm{M}$ & SA/hematoma & Lt hemiparesis & Confusion & Lt cSDH surgically treated & Recovery \\
\hline 54 & Probst, 1968, c1 & $33, \mathrm{M}$ & $\mathrm{Ac} / \mathrm{TBI}$ & Rt hemiplegia & Sz, rt mydriasis, HICP & $\begin{array}{l}\text { Rt frontal hematoma surgically } \\
\text { treated }\end{array}$ & Recovery \\
\hline 55 & Probst, 1968, c2 & $11, M$ & $\mathrm{Ac} / \mathrm{TBI}$ & Rt hemiparesis & Rt mydriasis, coma & $\begin{array}{r}\text { Rt frontal depressed fracture, \& } \\
\text { contusions surgically treated }\end{array}$ & Recovery \\
\hline 56 & Probst, 1968, c3 & $32, F$ & Chr/tumor & Lt hyperreflexia & Gerstmann syndrome & $\begin{array}{l}\text { Lt temporal astroblastoma surgi- } \\
\text { cally treated }\end{array}$ & Recovery \\
\hline 57 & Probst, 1968, c4 & $46, F$ & Chr/tumor & Lt hemiparesis & HICP, It ataxia & $\begin{array}{l}\text { Lt cerebellar hemangioblastoma } \\
\text { surgically treated }\end{array}$ & Recovery \\
\hline 58 & $\begin{array}{l}\text { Dupuis-Leveau, } \\
\text { 1979, c1 }\end{array}$ & $64, \mathrm{M}$ & SA/hematoma & Rt hemiparesis & Aphasia, S & Rt cSDH surgically treated & Recovery \\
\hline
\end{tabular}


» CONTINUED FROM PAGE 4

TABLE 1. Summary of data for the cohort of well-described patients with IH reported in the pre-MRI era (1858-1979)

\begin{tabular}{|c|c|c|c|c|c|c|c|}
\hline $\begin{array}{l}\text { Case } \\
\text { No. }\end{array}$ & Authors \& Year* & $\begin{array}{l}\text { Age (yrs), } \\
\text { Sex }\end{array}$ & $\begin{array}{l}\text { Time Onset/ } \\
\text { Etiology }\end{array}$ & Motor Deficit & $\begin{array}{c}\text { Additional Neurological } \\
\text { Symptoms }\end{array}$ & Pathological/Surgical Evidence & Outcome \\
\hline 59 & $\begin{array}{l}\text { Dupuis-Leveau, } \\
\text { 1979, c2 }\end{array}$ & $40, \mathrm{M}$ & SA/hematoma & Rt hemiparesis & No & Rt cSDH surgically treated & Recovery \\
\hline 60 & $\begin{array}{l}\text { Dupuis-Leveau, } \\
\text { 1979, c3 }\end{array}$ & $40, F$ & SA/hematoma & Lt hemiparesis & No & Lt cSDH surgically treated & Recovery \\
\hline 61 & $\begin{array}{l}\text { Dupuis-Leveau, } \\
\text { 1979, c4 }\end{array}$ & $36, F$ & SA/hematoma & Rt hemiparesis & Rt mydriasis & Rt cSDH surgically treated & Recovery \\
\hline 62 & $\begin{array}{l}\text { Dupuis-Leveau, } \\
\text { 1979, c5 }\end{array}$ & $47, M$ & SA/hematoma & Rt hemiparesis & Aphasia & Rt cSDH surgically treated & Recovery \\
\hline 63 & $\begin{array}{l}\text { Dupuis-Leveau, } \\
\text { 1979, c6 }\end{array}$ & $42, \mathrm{M}$ & Ac/hematoma & Rt hemiparesis & Lt mydriasis & Rt cSDH surgically treated & Recovery \\
\hline 64 & $\begin{array}{l}\text { Dupuis-Leveau, } \\
\text { 1979, c7 }\end{array}$ & $74, \mathrm{M}$ & SA/hematoma & Rt hemiparesis & Aphasia, coma & Rt cSDH surgically treated & Recovery \\
\hline 65 & $\begin{array}{l}\text { Dupuis-Leveau, } \\
\text { 1979, c8 }\end{array}$ & $64, \mathrm{~F}$ & Ac/hematoma & Lt hemiparesis & HICP, It CN III palsy, S & Lt cSDH surgically treated & Recovery \\
\hline 66 & $\begin{array}{l}\text { Dupuis-Leveau, } \\
\text { 1979, c9 }\end{array}$ & $72, \mathrm{M}$ & SA/hematoma & Lt hemiparesis & Sz, S & Lt cSDH surgically treated & Recovery \\
\hline 67 & $\begin{array}{l}\text { Dupuis-Leveau, } \\
\text { 1979, c10 }\end{array}$ & $60, F$ & C/hematoma & Rt hemiparesis & HICP, coma & Rt cSDH surgically treated & Recovery \\
\hline 68 & $\begin{array}{l}\text { Dupuis-Leveau, } \\
\text { 1979, c11 }\end{array}$ & $53, \mathrm{~F}$ & Ac/hematoma & Rt hemiparesis & Sz, S & Rt cSDH surgically treated & Death \\
\hline 69 & $\begin{array}{l}\text { Dupuis-Leveau, } \\
\text { 1979, c12 }\end{array}$ & $73, \mathrm{M}$ & SA/hematoma & Rt hemiparesis & Aphasia, S & Rt cSDH surgically treated & Death \\
\hline 70 & $\begin{array}{l}\text { Dupuis-Leveau, } \\
\text { 1979, c13 }\end{array}$ & $42, \mathrm{~F}$ & Ac/hematoma & Rt hemiparesis & Rt hemianopsia, S & Rt cSDH surgically treated & Recovery \\
\hline 71 & $\begin{array}{l}\text { Dupuis-Leveau, } \\
\text { 1979, c14 }\end{array}$ & $49, F$ & SA/hematoma & Lt hemiplegia & $S$ & Lt cSDH surgically treated & Recovery \\
\hline 72 & $\begin{array}{l}\text { Dupuis-Leveau, } \\
\text { 1979, c15 }\end{array}$ & $46, F$ & Ac/hematoma & Lt hemiparesis & $\mathrm{S}, \mathrm{Sz}$ & Lt cSDH surgically treated & Recovery \\
\hline 73 & $\begin{array}{l}\text { Dupuis-Leveau, } \\
\text { 1979, c16 }\end{array}$ & $28, M$ & SA/hematoma & Lt hemiparesis & HICP, S & Lt cSDH surgically treated & Recovery \\
\hline 74 & $\begin{array}{l}\text { Dupuis-Leveau, } \\
\text { 1979, c17 }\end{array}$ & $46, F$ & SA/hematoma & Rt hemiparesis & HICP, S & Rt cSDH surgically treated & Recovery \\
\hline 75 & $\begin{array}{l}\text { Dupuis-Leveau, } \\
\text { 1979, c18 }\end{array}$ & $38, F$ & Ac/hematoma & Rt hemiparesis & HICP, S & Rt cSDH surgically treated & Recovery \\
\hline
\end{tabular}

$\mathrm{Ac}=$ acute; $\mathrm{aSDH}=$ acute subdural hematoma; $\mathrm{BT}=$ bitemporal $; \mathrm{c}=$ case; $\mathrm{Chr}=$ chronic $\mathrm{CN}=$ cranial nerve; $\mathrm{CPA}=\mathrm{cerebellopontine} \mathrm{angle} ; \mathrm{CPL}=\mathrm{contralateral}$ peduncle incisura; cSDH = chronic subdural hematoma; CST = corticospinal tract; DTC = decompressive temporal craniectomy; EDH = epidural hematoma; GSW = greater sphenoid wing; $\mathrm{HICP}=$ symptoms/signs of high intracranial pressure; $\mathrm{NA}=$ not available; $\mathrm{S}=$ somnolence; $\mathrm{SA}=$ subacute; $\mathrm{SAH}=$ subarachnoid hemorrhage; $\mathrm{Sz}$ = seizures; $\mathrm{TBI}=$ traumatic brain injury; $\mathrm{X}=$ presence of pyramid decussation; $\mathcal{K}=$ absence of pyramid decussation.

* Lederhose (1896), Pineles (1896), Zenner (1898), Bernex (1904), Joltrain (1906), Burley (1906), Oppenheim (1906), and Clunet (1908) are cited in Binet (1909); Bonola (1916), Demole de Genève (1918), and Giannuli (1918) are cited in Couronne (1925); and Torkildsen (1937) is cited in Ectors (1945).

that was compatible with prior neuroanatomical studies by Paul Flechsig (1847-1929) demonstrating the variability in the degree of decussation of the pyramidal tract. ${ }^{11}$ The great respect for Charcot's theory led to a systematic search for abnormalities in the pyramidal tract decussation among IH patients, with contradictory results (Table 1).

\section{Ipsilateral Hemiparesis Caused by Functional Impairment of a Cerebral Region Distant From the Injury}

In parallel with Charcot's clinicoanatomical method, some neurologists defended the unorthodox view that many neurological deficits actually corresponded to functional disorders produced by the imbalance in the input connectivity on brain regions distant from the site initially injured. Two such closely related theories were proposed for IH.

\section{Theory of "Cerebral Irritation" From a Distance}

True to his eccentric and contentious nature, French physiologist and neurologist Charles-Édouard BrownSéquard (1817-1894) rejected Charcot's clinicoanatomical correlation method and proposed the original concept that $\mathrm{IH}$ represented the effect of an irritative action 
TABLE 2. Summary of data supporting each of the 4 main historical hypotheses conceived to explain IH

\begin{tabular}{llccc}
\hline \multicolumn{1}{c}{ Theory Variable } & $\begin{array}{c}\text { Absence of CST } \\
\text { Decussation }\end{array}$ & Callosal Interruption & $\begin{array}{c}\text { Contralateral Peduncle } \\
\text { Compression }\end{array}$ & $\begin{array}{c}\text { Contralateral Cerebral } \\
\text { Ischemia }\end{array}$ \\
\hline No. of cases (ancient \& modern series) & Scarce & Scarce & Numerous & Scarce \\
\hline Laterality/motor dominance & Independent & Dependent & Independent & Independent \\
\hline Mass effect & Unnecessary & Unnecessary & Necessary* & Necessary \\
\hline Location of supra/infratentorial (primary insult) & Independent & Dependent & Independent & Dependent \\
\hline Tentorial anthropometry & Independent & Independent & May play a role & Independent \\
\hline Motor deficit distribution & $\begin{array}{c}\text { Dependent on primary } \\
\text { lesion topography }\end{array}$ & $\begin{array}{c}\text { Dependent on primary } \\
\text { lesion topography }\end{array}$ & Crural & Dependent on vascular \\
territory involved & Independent & Independent \\
\hline Presence of other nervous system anomalies & Frequent & Independent & Indent & Numerous \\
\hline Evidence from modern ancillary tests & Absent & Absent & Absent \\
\hline
\end{tabular}

* Scarce cases of IH have been described in the literature, with MRI evidence of a contralateral peduncle lesion in the absence of significant mass effect, as described in the text and Fig. 6.
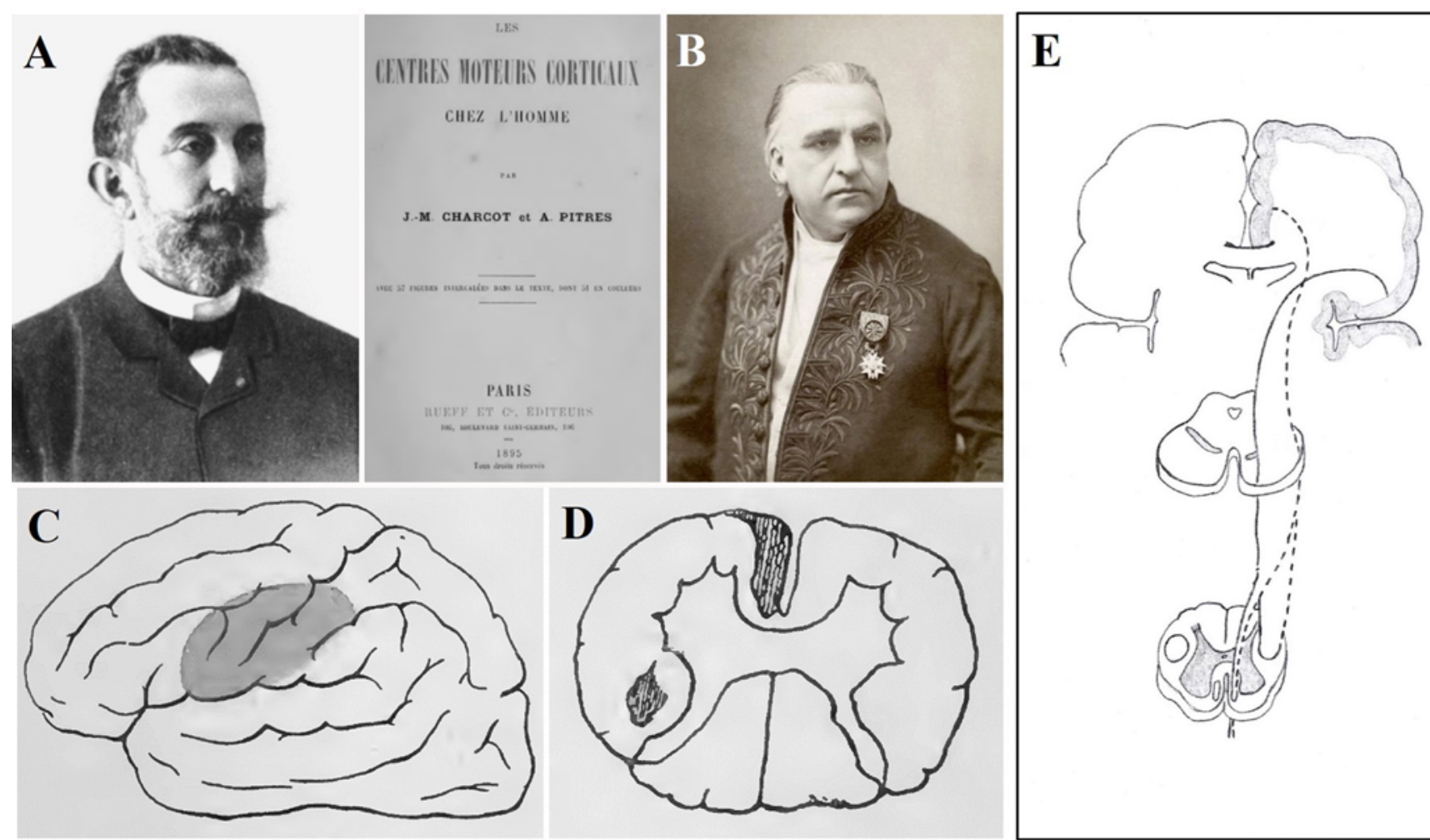

FIG. 1. Lack of decussation of the corticospinal tract theory. Albert Pitres (1848-1928) (A), shortly after the death of his mentor Jean-Martin Charcot (1825-1893) (B), published in 1895 the monograph entitled Motor Cortical Centers in Man (front page of the work, between panels A and B), based on the clinical and pathological analysis of 174 patients with cerebral lesions. In Chapter 10, addressing the subject of "Contradictory Observations," a patient with right hemiparesis was described. At his autopsy, a "ramollisement du cerveau," or cerebral infarct involving the peri-rolandic region and the base of the third frontal gyrus of the right hemisphere, was found (C), as were signs of secondary degeneration of the ipsilateral corticospinal tract at the spinal cord level (D). A schematic illustration (E) of the anatomical course followed by a nondecussated corticospinal tract. An injury of this tract at any level in its course would cause IH. Photographs of Pitres and Charcot: Reproduced from Wikimedia Commons (https://commons. wikimedia.org/wiki/File:Albert_Pitres.jpg) and the US National Library of Medicine website (http://resource.nlm.nih.gov/101425121), respectively. Public domain. $C$ and D: Reproduced from Charcot JM, Pitres A: Des observations contradictoires, in Charcot JM, Pitres A (eds): Les centres moteurs corticaux chez l'homme. Paris: Rueff et Cie Éditeurs, 1895, pp 183-191 (French) (http:// catalogue.bnf.fr/ark:/12148/cb31118881v). Public domain. 

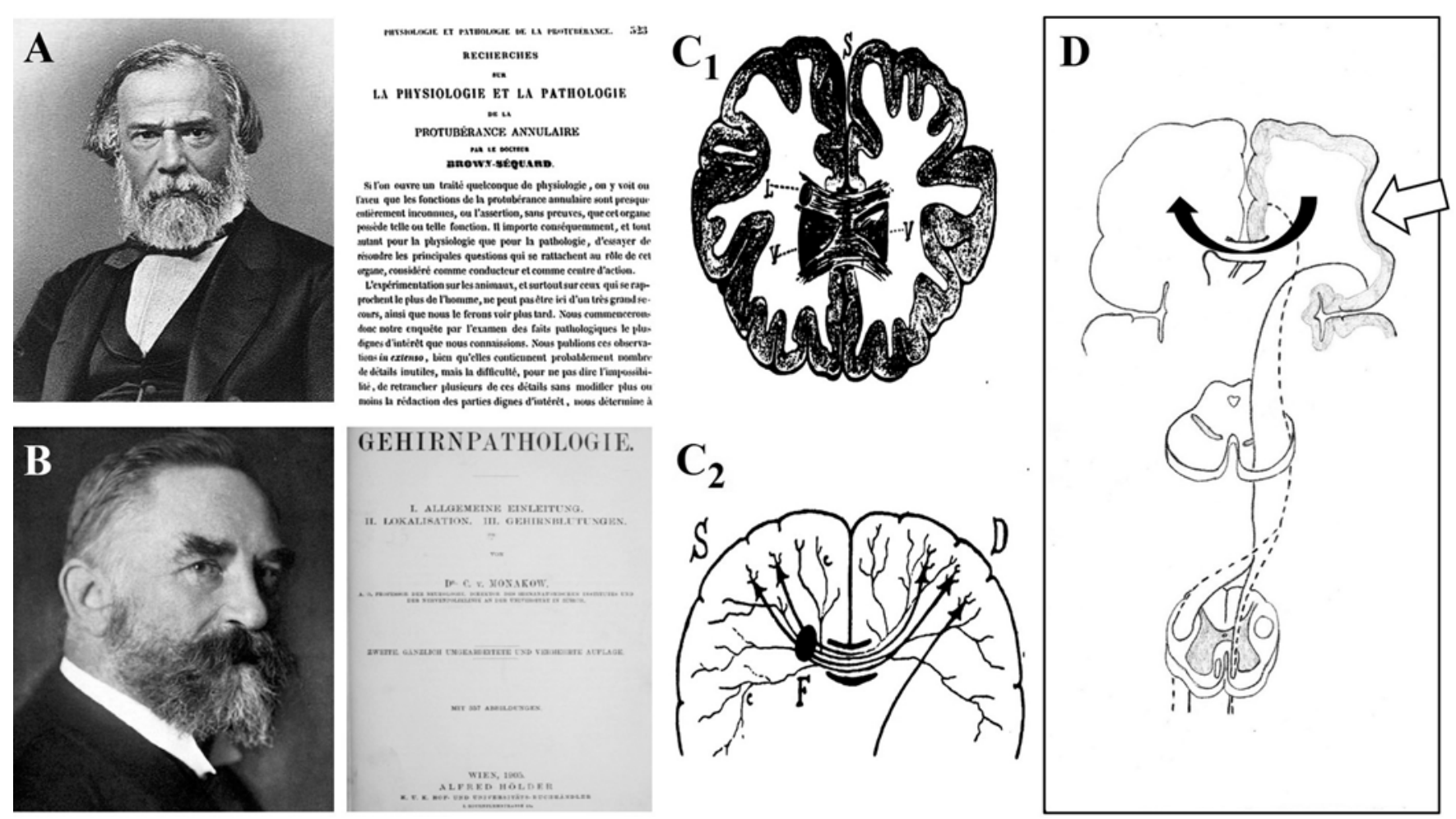

FIG. 2. Theory of cerebral dysfunction caused by a distant injury. A: Mauritian physiologist and neurologist Charles-Édouard Brown-Séquard (1817-1894) originally proposed this controversial concept in an article addressing the physiology and pathology of the pons. Text: Reprinted from Brown-Séquard CE: Recherches sur la physiologie et la pathologie de la protuberance annulaire. Journal de la Physiologie de l'Homme et des Animaux 1:523-539, 1858 (French). Public domain. B: Constantin von Monakow (1853-1930) published in 1905 the second edition of his monograph Gehirnpathologie, in which he explained for the first time his concept of diaschisis. Text: Reprinted from von Monakow C: Shock und diaschisis, in von Monakow C (ed): Gehirnpathologie. Vienna: Alfred Hölder, 1905, pp 236-248 (German). Public domain. $\mathbf{C}_{1}$ and $\mathbf{C}_{2}$ : Original illustrations of the paper published by Ascenzi in 1908 focused on the role of the corpus callosum in motor function. Reprinted from Ascenzi O: Una cisti emorragica del corpo calloso. Riv Patol Nerv Ment 13:1-15, 1908 (Italian). Public domain. Schematic representation of a hemorrhagic cystic lesion involving the left side of the genu of corpus callosum ( $\mathrm{L}$ in $\mathrm{C}_{1}, \mathrm{~F}$ in $\mathrm{C}_{2} ; \mathrm{S}=$ interhemispheric fissure, $\mathrm{V}=$ lateral ventricles). According to this author, the lesion would affect mostly the callosal fibers projecting toward the left hemisphere, grouped in more compacted bundles in the left precommissural region as opposed to the right side of the genu of corpus callosum ( $\mathrm{F}$ in $\mathrm{C}_{2} ; \mathrm{S}=1 \mathrm{left}$, $\mathrm{D}=$ right). Illustrative scheme (D) showing the concept of commissural diaschisis. A lesion affecting the primary motor cortical region within the dominant hemisphere (white arrow) causes functional impairment of a distant area in the nondominant hemisphere due to the interference it produces in the normal neuronal input from the dominant hemisphere through the corpus callosum (black curved arrow). Photographs of Brown-Séquard and von Monakow reproduced from the Bibliothèque Interuniversitaire de Santé Paris Descartes website (http://www.bium.univ-paris5.fr/images/livres/21950/0013.jpg) and Wikimedia Commons (https://commons.wikimedia.org/wiki/File:Monakov.PNG), respectively. Public domain.

on a certain cerebral area that originated from a distant damaged region or "irritation agissant à distance"" (Fig. 2A). Based on a prior IH case published by Antoine Joseph Jobert de Lamballe (1799-1867) of a patient with a voluminous supra- and infratentorial fifth cranial nerve schwannoma (Table 1, case 1), Brown-Séquard reasoned that this deficit could not be explained by the interruption of nerve flow at the compressed cerebral peduncle, as it should have caused contralateral hemiparesis. ${ }^{8}$ Rather, it would be the result of an "irritative" phenomenon originating in distant structures compressed by the tumor, such as the middle cerebellar peduncle and the cerebellum itself. Brown-Séquard assumed it was a trophic action of the cerebellum on the contralateral cerebral hemisphere, and therefore IH could be a cerebellum-mediated effect. $^{8}$

\section{Theory of Diaschisis}

The Russo-Swiss neuropathologist and physiologist Constantin von Monakow (1853-1930) addressed the paradoxical symptom of IH in his monumental monograph Gehirnpathologie (Brain Pathology). In line with BrownSéquard's view, von Monakow questioned both Charcot's theory of the absence of pyramidal tract decussation and the existence of mechanical compression of the contralateral corticospinal tract, concepts he felt lacked solid pathological evidence (Fig. 2B). ${ }^{44}$ In 1905, he developed the innovative, complex concept of "diaschisis" (from the Greek words dia and schizien, meaning "split in half") for unexplained transitory neurological deficits associated with localized injuries to the brain. ${ }^{26,44}$ Von Monakow believed that if a particular brain region was damaged, the loss of function could affect other distant parts of the brain not 

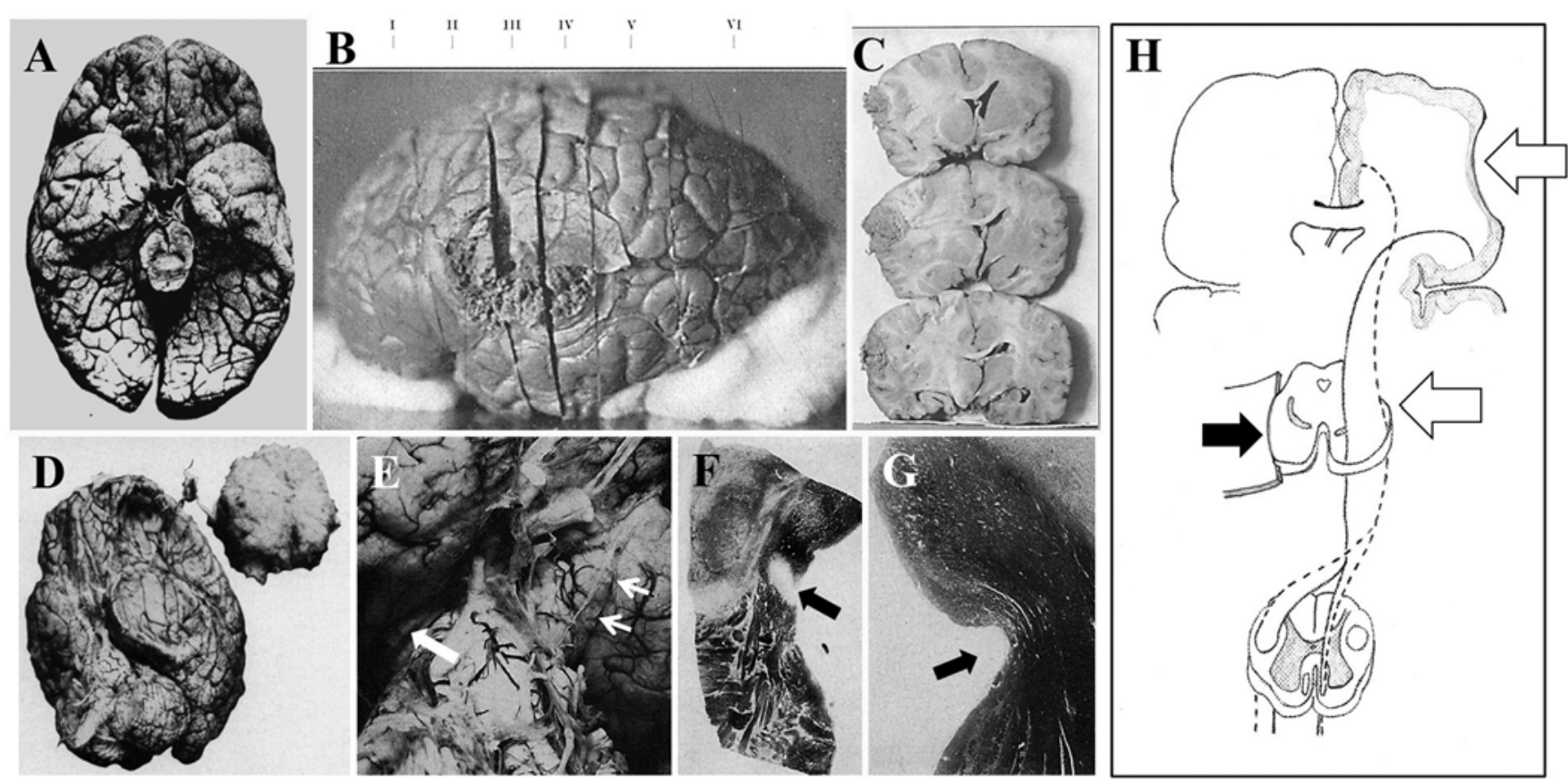

FIG. 3. Historical landmarks in the concept of notching of the contralateral cerebral peduncle as the origin of IH. A: Autopsy brain specimen showing the transtentorial descendent herniation of the mesial temporal lobe caused by a hemorrhagic metastasis expanding at the post-central gyrus of the right hemisphere. The herniation causes the mechanical deformation of the ipsilateral peduncle. Reprinted from Meyer A: Herniation of the brain. Arch Neurol Psychiatry 4:387-400, 1920. Public domain. B and C: Serial sections of the brain specimen studied by Groeneveld and Schaltenbrand, showing the brain distortion caused by an extraaxial tumor. Reproduced with permission from Springer Nature. From Groeneveld A, Schaltenbrand G: Ein Fall von Duraendotheliom über der Grosshirnhemisphäre mit einer bemerkenswerten Komplikation: Läsion des gekreuzten Pespedunculidurch Druckauf den Rand des Tentoriums. Dtsch Z Nervenheilkd 97:32-50, 1927 (German). The authors described the existence of a structural lesion involving the contralateral peduncle in this specimen, but no pictorial evidence was provided in their article. D-F: Pictures of the brain specimen autopsied and reported by Kernohan and Woltman. Reproduced from Kernohan JW, Woltman HW: Incisura of the crus due to contralateral brain tumor. Arch Neurol Psychiatry 21:274-287, 1929. Copyright American Medical Association. The mass effect of an endothelioma (meningioma) on the brain bulk (D) is shown, as are the ipsilateral uncal herniation (short arrows in $\mathrm{E}$ ) and the notching of the contralateral cerebral peduncle (long arrow in E, with an underlying focal destruction of nervous tissue and myelin sheathing of the corticospinal tract). G: In a later report by the same researchers, a peduncular notch without structural damage of the underlying nervous tissue was demonstrated (arrow). Reproduced from Kernohan JW, Woltman HW: Incisura of the crus due to contralateral brain tumor. Arch Neurol Psychiatry 21:274-287, 1929. Copyright American Medical Association. H: Illustrative schematic showing the mechanical compression of the contralateral peduncle against the free tentorial edge (black arrow) secondary to the lateral displacement of the brainstem caused by a supratentorial or infratentorial mass (white arrows).

seemingly related to the site of injury. Therefore, diaschisis should be interpreted as a transitory functional impairment caused by the temporary blocking of normal input on a particular brain region anatomically linked to the distant injured site. ${ }^{26,44}$ If the injury was not too severe, functional behavior would recover once the period of diaschisis wore off. The specific paradoxical case of IH would represent, according to von Monakow, an example of "diaschisis corticommisuralis" caused by impairment of the normal functional activation of the contralateral hemisphere by fibers coming from the dominant hemisphere through the corpus callosum, a hypothesis supported by the findings published by Ascenzi in 1908 (Fig. $2 \mathrm{C}_{1}$ and $\mathrm{C}_{2}$ ). ${ }^{3}$

\section{Compression of the Contralateral Corticospinal Tract}

The most widely accepted theory for explaining IH was the compression of the contralateral corticospinal tract at any level along its course above the pyramidal decussation. ${ }^{6,14,34}$ Several different tract compression mechanisms were proposed in the 19th century, among them the flattening ("aplatissement") of the motor cortex against the inner skull surface, the compression of the tract secondary to hydrocephalus ("hydropsie"), the development of swelling in the contralateral hemisphere, and/or the coexistence of disturbances in cerebral blood flow. ${ }^{21}$ However, the major breakthrough occurred in 1920, when the neuropathologist and psychiatrist Adolf Meyer (1866-1950) demonstrated the dynamic displacements caused by intracranial mass lesions on the cerebral soft tissue (Fig. 3A). ${ }^{35}$ Arguably, the recognition of brain herniations proved the most relevant contribution to the origin of the false localizing signs associated with intracranial lesions. The seminal concepts of "contralateral peduncular notch" and the "syndrome of the third frontal convolution" (or Ectors' syndrome) substantially improved insight into the way IH may occur in association with brain herniation.

\section{Kernohan's Notch Phenomenon}

In 1905 German neurologist Albert Knapp recognized 
hemiparesis alternans, or motor deficit of the ipsilateral oculomotor nerve and contralateral hemiparesis, as a clinical manifestation typical of temporal lobe tumors that compress the ipsilateral cerebral peduncle through uncal herniation. ${ }^{32}$ Knapp was aware of the occurrence of $\mathrm{IH}$ in this clinical setting, and he was the first to attribute this paradoxical finding to mechanical compression of the contralateral cerebral peduncle. ${ }^{32}$ The pathological evidence for this mechanism was not reported until the end of the 1920s, when two groups of researchers-the Dutch and German neurologists Arnold Groeneveld (1895-1962) and Georges Schaltenbrand (1897-1979), respectively, ${ }^{28}$ and the American neuropathologist James W. Kernohan (1896-1981) and neurologist Henry W. Woltman (1889-1964) rately published the evidence of mechanical injury at the cerebral peduncle presumably caused by its compression against the free edge of the cerebellar tentorium (Fig. 3). ${ }^{16}$ Encouraged by this finding, Kernohan and Woltman carried out systematic research on brains obtained from Mayo Clinic autopsies in which they identified the presence of such an indentation or notch in the contralateral cerebral peduncle in 34 of 276 brains from patients with intracranial neoplasms. ${ }^{31}$ This 1929 study had such a positive impact on the scientific community that $\mathrm{IH}$ was henceforth renamed the "Kernohan-Woltman notch phenomenon" or simply "Kernohan's notch phenomenon" (Fig. 3). Perhaps the most striking finding of their study was that 13 patients with a peduncular notch did not show clinical hemiparesis or signs of corticospinal tract impairment such as hyperreflexia or a Babinski sign, a fact for which Kernohan and Woltman were unable to provide a proper explanation. ${ }^{31}$ This inconsistency has been used to support the theory of diaschisis by its proponents. ${ }^{17-19}$

\section{Ectors' Syndrome: A Particular Type of Kernohan's Notch Caused} by Mass Effect on the Third Frontal Convolution

The next milestone in the elucidation of $\mathrm{IH}$ causes was the identification by the Belgian neurosurgeon Léon Ectors of a particular clinical syndrome associated with meningiomas involving the third or inferior convolution of the frontal lobe (Fig. 4A and B). Supported by ample and convincing clinical, experimental, and bibliographic evidence, Ectors was able to categorize the fundamental groups of symptoms and/or signs in a monograph hardly mentioned in medical literature. ${ }^{22}$ Among symptoms such as mental disturbances, conjugated cephalic and ocular rotation toward the site of the tumor, due to impairment of Brodmann area 8, and those typical of high intracranial hypertension, Ectors included what he called "early ipsilateral hemiparesis" as a specific neurological finding associated with meningiomas of the third frontal lobe gyrus. Ectors' syndrome also included typical radiological findings such as angiographic ingurgitation of the anterior branch of the middle meningeal artery and contralateral displacement of cerebral ventricles observed in air ventriculography. ${ }^{22-24}$

Several ancient reports of IH seem to fit in Ectors' syndrome (Table 1). According to Ectors, IH severity would not depend directly on the size of the tumor, but rather it would be proportional to the degree of lateral displacement of the brainstem. ${ }^{22-24}$ Long-term expanding lesions growing at the basal frontal lobe would cause an optimal compressive force vector across the brain tissue, pointing to the tentorial hiatus and the brainstem, therefore increasing the likelihood of the contralateral peduncle's lateral displacement against the free edge of the tentorium, even in the absence of uncal herniation (Fig. 4C-E). Typically, IH would develop slowly, following a leg-arm-face involvement sequence as a result of the somatotopic distribution of corticospinal bundles within the cerebral peduncle. ${ }^{22}$ Ectors remarked that in the early stages of the disease, the only manifestation might be a mild hyperreflexia in the leg, which can be easily overlooked. ${ }^{22-24}$ The characteristic full reversibility of hemiparesis when the intracranial mass effect was promptly alleviated suggests that the major factor contributing to IH in Ectors' syndrome is the elastic deformation of the contralateral peduncle against the tentorial free edge, without structural damage to the nervous tissue, as observed by Kernohan and Woltman. ${ }^{31}$

\section{Cerebrovascular Dysfunction}

In the late 1970s Géraldine Dupuis-Leveau from Reims proposed hypoperfusion of the contralateral hemisphere as a complementary physiopathological mechanism for $\mathrm{IH}$, which would act together with contralateral peduncle compression. $^{21}$ As part of her doctoral thesis, Dupuis-Leveau analyzed the cerebral angiograms of 18 patients with IH included in a cohort of 180 cases of surgically treated subdural hematomas and observed a reduced cerebral blood flow in these patients, a finding she correlated with kinking and mechanical flattening of the carotid artery contralateral to the hematoma against the posterior clinoid process and the petroclinoid ligament. ${ }^{21}$ Characteristically, the combined displacement of both carotid arteries gives rise to a "windshield wiper" (from the French en essuieglaces) aspect of these vessels in the anteroposterior angiographic projection (Fig. 5). As a result, either a predominating brachiofacial (ischemia of the perisylvian region) or crural motor (ischemia of the pericallosal area) deficit can occur, depending on the carotid artery branches that develop reduced blood flow. ${ }^{21}$ This vascular mechanism suggests that early surgical evacuation of the hematoma guarantees complete clinical recovery, provided that cerebral hypoperfusion did not reach the threshold for irreversible ischemic injury.

\section{Ipsilateral Hemiparesis: Past, Present, and Future}

Fueled by modern neuroradiological techniques, IH has received renewed interest in recent years. Even so, it remains an exceptional clinical finding. Indeed, only 39 cases published since 1991 could be collected in the most recent comprehensive review on this subject. ${ }^{45}$ Léon Ectors prophetically expressed his belief that IH would remain an underdiagnosed condition, especially in its early stages, unless a thorough neurological examination was carried out. ${ }^{22}$ Hence, subtle neurological findings suggestive of $\mathrm{IH}$, such as an unexplained muscle tone increase or the presence of abnormal reflexes, are rarely investigated when no motor deficit is observed. As evidence of this, most IH cases reported in the last 2 decades do not provide detailed neurological findings. ${ }^{45}$

Very little conceptual progress regarding this paradox- 

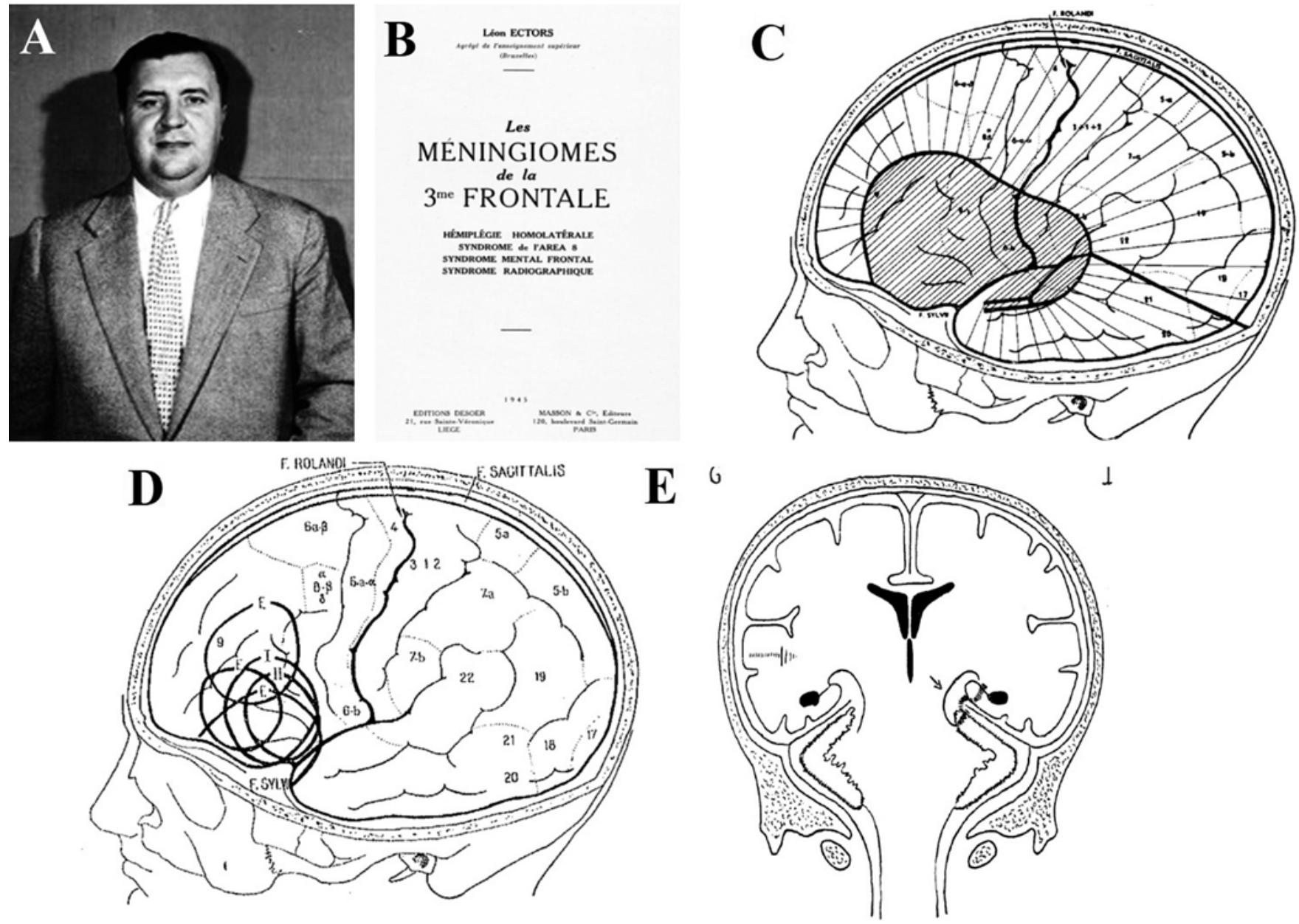

FIG. 4. A: Belgian neurosurgeon Léon Ectors photographed at the 4th International Neurological Congress held in Paris in 1949. Image courtesy of the US National Library of Medicine (http://resource.nIm.nih.gov/101414225). Published with permission. B: In 1945, Ectors included ipsilateral or paradoxical hemiparesis due to compression of the contralateral peduncle as a symptom characteristic of meningiomas that developed at the third frontal gyrus. C: Illustrative scheme showing the anatomical relationships of the encephalon with the falx and the cerebellar tentorium; the projection of the third frontal gyrus on the falx hiatus is shown. $\mathrm{D}$ and $\mathrm{E}$ : A solid large tumor like a meningioma expanding at this site would generate a force vector optimal enough to displace the contralateral peduncle against the free tentorial edge, without producing a concomitant uncal herniation (arrow in E). B to E: Reproduced from Ectors L (ed): Les méningiomes de la troisiéme frontale. Paris: Masson, 1945 (French), available at Université Libre de Bruxelles website (https://difusion.ulb.ac.be/vufind/Record/ULB-DIPOT:oai:dipot.ulb.ac.be:2013/215987/Holdings). Published with permission.

ical phenomenon has been made by using current diagnostic methods, as most of the anatomical and functional hypotheses proposed for IH throughout history have not been adequately checked or tested. In principle, it seems reasonable to discard the absence of corticospinal tract decussation, as there has been no case of IH reported in modern literature confirming such a finding. ${ }^{45}$ In the cohort of well-described IH cases in the MRI era (1990-2019), IH is predominantly reported among brain-injured patients, in contrast to earlier descriptions involving slowly growing intracranial mass lesions, such as meningiomas and chronic subdural hematomas (Table 1). ${ }^{45}$ In those reports of patients with a rapidly expanding supratentorial massepidural or acute subdural hematoma-the clinical picture is largely dominated by a reduced level of consciousness, in addition to a variable motor impairment affecting the 4 limbs in most severe cases. ${ }^{45}$ This type of $\mathrm{IH}$ was referred to as "late ipsilateral hemiparesis" by Ectors and is usually associated with clinical signs of severe brainstem dysfunction., ${ }^{9,22}$ The initial diagnostic tool usually employed in these cases is cranial CT, which does not allow for an optimal assessment of the brainstem. Nevertheless, in the face of a persistent IH after surgical decompression, MRI studies were obtained that proved conclusively the presence of Kernohan's notch phenomenon as the cause of this symptom in most cases (Fig. 6A-C).$^{45}$ In these studies, a rounded lesion involving the contralateral cerebral peduncle was usually observed, typically hyperintense on T2-weighted and FLAIR sequences and hypointense on T1-weighted sequences, with or without hemosiderin deposits on the T2-weighted gradient-echo sequence. ${ }^{9}$ Diffusion-weighted imaging seems to be the more sensitive sequence for the detection of the early brainstem alterations associated with Kernohan's notch. ${ }^{45}$ A characteristic 


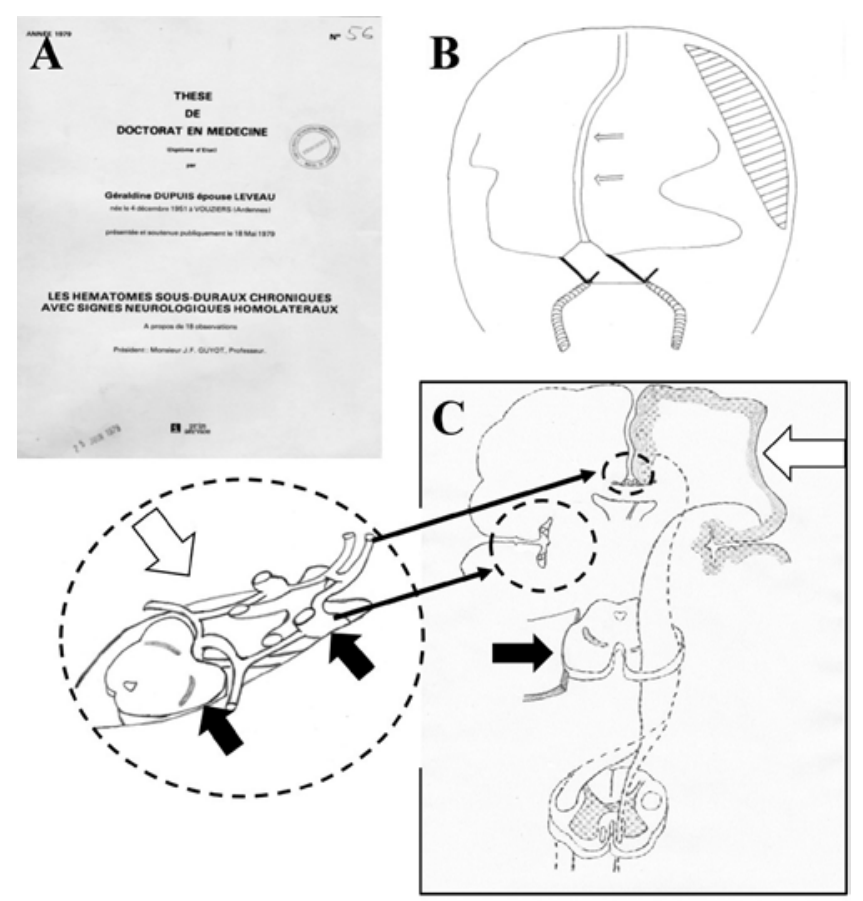

FIG. 5. A: Front page of Dupuis-Leveau's doctoral dissertation. B: Schematic representation of the "windshield wiper" image, observed in the anteroposterior projection of a cerebral angiogram, caused by vascular displacement of carotid arteries secondary to a subdural hematoma. C: The brain displacement (white arrows) would cause compression of the contralateral cerebral peduncle against the free tentorial edge, as well as the kinking of the contralateral internal carotid artery against rigid structures at the cranial base (black arrows), giving rise to cerebral ischemia of the contralateral hemisphere involving the regions supplied by the median cerebral and/or anterior cerebral arteries. A and B: Reproduced from Dupuis-Leveau G: Les hematomes sousduraux chroniques avec signes neurologiques homolateraux. A propos de 18 observations [doctoral thesis]. Faculty of Medicine, Reims, 1979 (French). Published with permission.

hyperintense signal within the cerebral peduncle owing to the restriction in the apparent diffusion coefficient (ADC) map during the acute stage of this phenomenon probably reflects the presence of cytotoxic edema, as it attenuates or disappears in control studies. ${ }^{42}$ Partial or total recovery from motor deficit, even in patients in whom a complete disruption of a normally decussated corticospinal tract has been demonstrated using diffusion tensor imaging, ${ }^{29,33,37}$ can be explained by the resolution of cytotoxic edema beneath the peduncle notch. This concept is supported by Kernohan and Woltman's original histological studies confirming the disruption of myelin sheaths at the level of the injured area behind the notch, whereas most of the corresponding axons of the corticospinal tract were spared from destruction. ${ }^{31}$

A second subgroup of recent IH reports has shown examples of the clinical picture that Ectors characterized as early $\mathrm{IH},{ }^{22}$ in which the slow progressive growth of the intracranial mass lesion permitted a cerebral MRI study before the surgical procedure. ${ }^{45}$ These studies evidenced the notching of the contralateral cerebral peduncle against the free tentorial edge, with or without underlying structural damage to the corticospinal tract, which plausibly depends on the degree and duration of the compression (Fig. 6D and E).

Especially disconcerting are the few modern cases of IH in which no significant intracranial mass effect has been found. To increase the controversy, some of the authors of these cases have provided a radiological demonstration of the typical lesion in the contralateral peduncle described above, which has been interpreted by some authors as a traumatic contusion of this anatomical structure against the tentorial edge due to a swaying effect on the brainstem (Fig. 6F-H) ${ }^{5,25}$ Doubtful cases may benefit from the demonstration of a functional impairment of the contralateral corticospinal tract-using motor evoked potentials with transcranial magnetic stimulation-to support the clinical diagnosis,,${ }^{5,10}$ although this finding does not completely rule out the hypothesis of diaschisis. ${ }^{18}$ Despite the fact that this concept still receives support from some neurologists, ${ }^{17-19}$ diaschisis in IH patients remains an unproven theory until further functional/metabolic investigation can verify it, ${ }^{26}$ as this hypothesis cannot properly account for either $\mathrm{IH}$ cases caused by lesions involving the nondominant hemisphere or those produced by infratentorial masses directly pushing the brainstem against the tentorium.

In order to explain the low rate of paradoxical hemiparesis and the physiopathological mechanism involved in unsolved cases, some authors have proposed the existence of some predisposing factors, such as the coexistence of a narrow tentorial incisure facilitating Kernohan's notch phenomenon or an insufficient blood supply to the contralateral brain hemisphere, especially in patients with atherosclerosis (Dupuis-Leveau's hypothesis). ${ }^{9} 21$ Yet, no definite conclusions regarding the effect of these variables can be stated, as very few studies include accurate measurements of the tentorial incisure, and no exhaustive MRI studies analyzing the vascular supply and brain perfusion have been performed in $\mathrm{IH}$ patients to date..$^{45}$

\section{Conclusions}

IH represents a still underdiagnosed paradoxical phenomenon that challenges the neurological paradigm for the hemispheric localization of cerebral motor functions. Even though modern neuroradiological and neurophysiological methods largely support Kernohan and Woltman's concept of contralateral peduncle notching against the free tentorial edge, none of the physiopathological mechanisms proposed throughout history can satisfactorily account for the cause of IH in all patients. To overcome this shortcoming, it will be essential to perform a thorough neurological examination, in addition to the most complete neuroradiological and neurophysiological investigations, including diffusion tract imaging, functional and perfusion MRI, anatomical measurements of the tentorial incisure, and motor evoked potential monitoring.

\section{Acknowledgments}

We wish to express our gratitude to George Hamilton for his critical review of the language and style of the manuscript. We also wish to thank Prof. Alfonso Villén Carrasco, who translated the consulted articles written in German. We are indebted to the staff at Ramón y Cajal University Hospital Medical Library (Madrid), the Bibliothèque Interuniversitaire de Santé (Université 

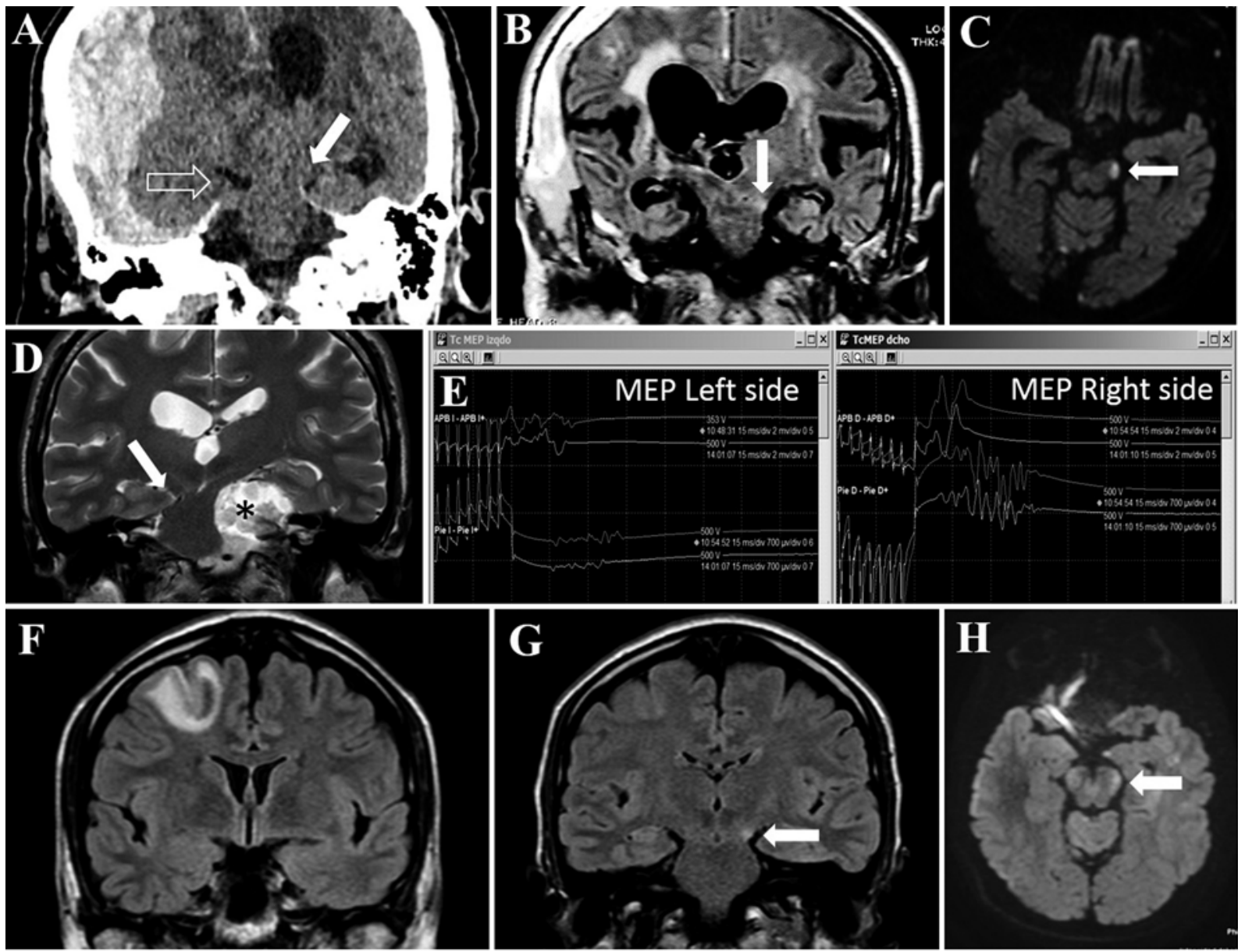

FIG. 6. Physiopathological mechanisms for IH examined using modern neuroradiological and neurophysiological methods in original cases from our own series. A-C: A 77-year-old male patient presented with signs of cerebral decerebration and a Glasgow Coma Scale score of 4 after suffering a severe traumatic brain injury. A: Coronal section of a cranial CT scan obtained at his admission showing a large acute subdural hematoma causing a marked displacement of the brain with signs of ipsilateral uncal herniation (hollow arrow) and compression of the contralateral peduncle against the tentorial edge (solid arrow). After a decompressive craniectomy and evacuation of the hematoma, the patient recovered normal consciousness but right spastic hemiparesis (grade 2/5) ipsilateral to the hematoma persisted. B and C: Brain MR images obtained postoperatively showing a hyperintense signal in the contralateral peduncle, right beneath the level of contact with the tentorial edge (arrows), in both the FLAIR (B) and the diffusion-weighted $(C)$ sequences, with restricted water diffusion in the ADC map. D and E: Patient with a left motor deficit involving predominantly the lower limb. D: MR image showing a supra/infratentorial left epidermoid cyst (asterisk) displacing the brainstem and causing indentation (arrow) of the contralateral peduncle against the free tentorial edge, without underlying signal alteration on the T2-weighted sequence. During resection of this lesion, motor evoked potentials (MEP) monitoring using electric transcranial stimulation showed delayed and reduced amplitude of motor responses in the left arm and leg (left and right panels in E). F-H: Images of a 41-year-old male patient who suffered cranial trauma secondary to a syncope. After recovering consciousness, he experienced seizures, time-space disorientation, gait imbalance, and hiccups. While a cranial CT scan only showed a frontal lobe contusive focus, MRI demonstrated a slight hyperintense signal at the contralateral peduncle (arrows) on the FLAIR image $(G)$ and diffusion-weighted sequence $(H)$, with water flow restriction on ADC maps. Note the lack of brainstem displacement. Remarkably, this patient did not show any motor deficits, and the effect of the peduncle lesion could only be correlated with an increased muscle tone accompanied by hyperreflexia in the thorough neurological examination.

Paris Descartes), and the Francisco de Vitoria University Library (Madrid) for their invaluable help in obtaining some of the original research material used for this study.

\section{References}

1. Achslogh J, Macken J: [A new case of meningioma of the third frontal convolution.] Acta Neurol Psychiatr Belg 59:163-173, 1959 (French)

2. Ardito R: Ematoma sottodurale della convessità con sín- drome piramidale omolaterale. Contributo clínico. Riv Patol Nerv Ment 78:123-154, 1954

3. Ascenzi O: Una cisti emorragica del corpo calloso. Riv Patol Nerv Ment 13:1-15, 1908

4. Babinski J, Clunet J: Tumeurs méningées unilatérales. Hémiplégie siègeant du même côté que les tumeurs. Rev Neurol (Paris) 16:707-709, 1908

5. Binder DK, Lyon R, Manley GT: Transcranial motor evoked potential recording in a case of Kernohan's notch syndrome: case report. Neurosurgery 54:999-1003, 2004 
6. Binet E: Contribution à l'étude des hémiplégies homolatérales: a propos d'un cas d'abscès de cerveau d'origine otique [doctoral thesis]. Paris: Faculty of Medicine, 1909

7. Blaise H: Deux cas peu conformes aux localisations cérébrales. Bull Soc Anat Paris 7:387-392, 1882

8. Brown-Séquard CE: Recherches sur la physiologie et la pathologie de la protuberance annulaire. Journal de la Physiologie de l'Homme et des Animaux 1:523-539, 1858

9. Carrasco R, Pascual JM, Navas M, Martínez-Flórez P, Manzanares-Soler R, Sola RG: Kernohan-Woltman notch phenomenon caused by an acute subdural hematoma. J Clin Neurosci 16:1628-1631, 2009

10. Carrasco-Moro R, Abreu-Calderón F, de Blas-Beorlegui G, Pascual JM, Ley-Urzaiz L: [Kernohan-Woltman notch phenomenon.] Rev Clin Esp 214:e97-e99, 2014 (Spanish)

11. Charcot JM, Pitres A: Des observations contradictoires, in Charcot JM, Pitres A (eds): Les centres moteurs corticaux chez l'homme. Paris: Rueff et Cie Éditeurs, 1895, pp 183191

12. Claude H, Vincent C, Lèvy-Valensi J: De l'hémiplégie homolatérale dans les tumeurs cérébrales. Rev Neurol (Paris) 20:612-614, 1910

13. Collier J: The false localising signs of intracranial tumour. Brain 27:490-508, 1904

14. Couronne M: Les hémiplégies homolatérales [doctoral thesis]. Paris: Faculty of Medicine, 1925

15. Couty M: Tumeur du pédoncule inférieur gauche. C R Seances Memoires Soc Biol 29:234-235, 1877

16. Dammers R, Volovici V, Kompanje EJ: The history of the Kernohan notch revisited. Neurosurgery 78:581-584, 2016

17. Derakhshan I: Kernohan notch. J Neurosurg 100:741-742, 2004 (Letter) (Erratum in J Neurosurg 101:721, 2004)

18. Derakhshan I: The Kernohan-Woltman phenomenon and laterality of motor control: fresh analysis of data in the article "Incisura of the crus due to contralateral brain tumor". J Neurol Sci 287:296, 2009 (Letter)

19. Derakhshan I: Transcranial motor evoked potential recording in a case of Kernohan's notch syndrome: case report. Neurosurgery 56:E1166, 2005 (Letter)

20. Dupré E, Camus P: Hémiplégie homolatérale gauche chez un débile gaucher, ancien hémiplégique infantile droit. Rev Neurol (Paris) 13:322-325, 1905

21. Dupuis-Leveau G: Les hematomes sous-duraux chroniques avec signes neurologiques homolateraux. A propos de 18 observations [doctoral thesis]. Reims, France: Faculty of Medicine, 1979

22. Ectors L (ed): Les méningiomes de la troisiéme frontale. Paris: Masson, 1945

23. Ectors L, Achslogh J: [The early homolateral pyramidal syndrome and meningiomas of the third frontal convolution.] Neurochirurgie 5:388-400, 1959 (French)

24. Ectors L, Heernu V: Contribution à l'étude des méningiomes de la troisième frontale. J Belge Neurol Psychiatr 8:497507, 1946

25. Eesa M, Bell K: Kernohan-Woltman notch phenomenon. J Trauma 69:1634, 2010

26. Finger S, Koehler PJ, Jagella C: The Monakow concept of diaschisis: origins and perspectives. Arch Neurol 61:283-288, 2004

27. Flatau E: De la radiothérapie des tumeurs du cerveau et de la moelle. Rev Neurol (Paris) 1:23-40, 1924

28. Groeneveld A, Schaltenbrand G: Ein Fall von Duraendotheliom über der Grosshirnhemisphäre mit einer bemerkenswerten Komplikation: Läsion des gekreuzten Pespedunculidurch Druckauf den Rand des Tentoriums. Dtsch Z Nervenheilkd 97:32-50, 1927

29. Jang SG, Pyun SB: Diffusion tensor tractography in two cases of Kernohan-Woltman notch phenomenon. Ann Rehabil Med 37:879-885, 2013
30. Kernohan JW, Woltman HW: Incisura of the crus due to contralateral brain tumor. Proc Staff Meetings Mayo Clinic 3:69-70, 1928

31. Kernohan JW, Woltman HW: Incisura of the crus due to contralateral brain tumor. Arch Neurol Psychiatry 21:274-287, 1929

32. Knapp A: Die tumoren des Schläfenlappens. Z Ges Neurol Psych 42:226-289, 1918

33. Mansilla-Fernández B, Isla-Guerrero A, Giner J, Royo-Orejas A: [Tractography in Kernohan's phenomenon: report of a case of acute subdural haematoma.] Rev Neurol 60:286-287, 2015 (Spanish)

34. Marie P: Hémiplégie collatérale, in Bruardel P, Gilbert A (eds): Traité de Médecine et de Thérapeutique. Paris: Librairie JB Baillière et Fils, 1901, Vol 8, pp 474-475

35. Meyer A: Herniation of the brain. Arch Neurol Psychiatry 4:387-400, 1920

36. Obrador Alcalde S: Sobre los falsos síntomas neurológicos de localización en los tumores supratentoriales. Actas Luso Esp Neurol Psiquiatr 7:183-195, 1948

37. Oh SI, Kim MJ, Oh KP, Kim HY, Kim SH, Kim HJ: Tractography of persistent ipsilateral hemiparesis following subdural hematoma. Can J Neurol Sci 40:601-602, 2013

38. Peyser E, Doron Y: Ipsilateral hemiplegia in supratentorial space occupying lesions. Int Surg 45:689-695, 1966

39. Pic M: Hémiplégie droite complète; autopsie: hémorragie uniquement localisée au lobe occipital droite. Lyon Med 107:560-561, 1906

40. Probst C: [Ipsilateral hemiparesis.] Schweiz Med Wochenschr 98:1793-1795, 1968 (German)

41. Purves-Stewart J (ed): Intracranial Tumors and Some Errors in Their Diagnosis. Edinburgh: Oxford University Press, 1927

42. Uesugi S, Suehiro E, Nakayama H, Suzuki M: Diffusionweighted magnetic resonance imaging in a case of Kernohan's notch phenomenon. Acta Neurochir (Wien) 152:18091810,2010

43. Vanhenverswyn: Pachyméningite cérébrale hémorragiquecontracture du côté de 1'épanchement. Bull Soc Anatomoclinique Lille 1:128, 1888

44. von Monakow C: Shock und diaschisis, in Von Monakow C (ed): Gehirnpathologie. Vienna: Alfred Hölder, 1905, pp 236-248

45. Zhang CH, DeSouza RM, Kho JS, Vundavalli S, Critchley G: Kernohan-Woltman notch phenomenon: a review article. Br J Neurosurg 31:159-166, 2017

\section{Disclosures}

The authors report no conflict of interest concerning the materials or methods used in this study or the findings specified in this paper.

\section{Author Contributions}

Conception and design: Carrasco-Moro, Pascual. Acquisition of data: all authors. Analysis and interpretation of data: CarrascoMoro, Pascual. Drafting the article: Carrasco-Moro, CastroDufourny, Pascual. Critically revising the article: Carrasco-Moro, Martínez-San Millán, Cabañes-Martínez, Pascual. Approved the final version of the manuscript on behalf of all authors: CarrascoMoro. Statistical analysis: Carrasco-Moro. Administrative/techni$\mathrm{cal} /$ material support: Martínez-San Millán, Cabañes-Martínez. Study supervision: Carrasco-Moro.

\section{Correspondence}

Rodrigo Carrasco-Moro: Ramón y Cajal University Hospital, Madrid, Spain.rocamo@gmail.com. 